\title{
An Attempt for an Emergent Scenario with Modified Chaplygin Gas
}

\author{
Sourav Dutta, Sudeshna Mukerji, and Subenoy Chakraborty \\ Department of Mathematics, Jadavpur University, Kolkata, West Bengal 700032, India \\ Correspondence should be addressed to Sourav Dutta; sduttaju@gmail.com
}

Received 17 December 2015; Accepted 21 February 2016

Academic Editor: Ahmed Farag Ali

Copyright ( 2016 Sourav Dutta et al. This is an open access article distributed under the Creative Commons Attribution License, which permits unrestricted use, distribution, and reproduction in any medium, provided the original work is properly cited. The publication of this article was funded by SCOAP S $^{3}$

\begin{abstract}
The present work is an attempt for emergent universe scenario with modified Chaplygin gas. The universe is chosen as spatially flat FRW space-time with modified Chaplygin gas as the only cosmic substratum. It is found that emergent scenario is possible for some specific (unrealistic) choice of the parameters in the equation of state for modified Chaplygin gas.
\end{abstract}

\section{Introduction}

The origin of the universe is a controversial issue in cosmology. It may start from the big bang singularity or there are proposals for nonsingular model of the universe. The inability of Einstein's general theory of relativity at zero volume leads to the well known big bang singularity in standard cosmology. To overrule this initial discomfortable situation various cosmological scenarios have been proposed and are classified as bouncing universes or the emergent universes. Here, we will choose the second option which results from searching for singularity-free inflationary scenario in the background of classical general relativity. In a word, emergent universe is a model universe, ever existing with almost static behavior in the infinite past $(t \rightarrow-\infty)$ (gradually evolves into inflationary stage) and having no time-like singularity. Also, the modern and extended version of the original Lemaitre-Eddington universe can be identified as the emergent universe scenario.

Long back in 1967, Harrison [1] showed a model of the closed universe containing radiation, which approaches the state of an Einstein static model asymptotically (i.e., $t \rightarrow$ $-\infty)$. This kind of model was again reinvestigated after a long gap by Ellis and collaborators [2,3]. Although they were not able to obtain exact solutions, they presented closed universes with a minimally coupled scalar field $\phi$ having typical self-interacting potential and possibly some ordinary matter with equation of state $p=w \rho,(-1 / 3 \leq w \leq 1)$, whose behavior similar to that of an emergent universe was highlighted. Then, in starobinsky model, Mukherjee et al. [4] derived solutions for flat FRW space-time having emergent character in infinite past. Subsequently, Mukherjee and associates [5] presented a general framework for an emergent universe model with an ad hoc equation of state connecting the pressure and density, having exotic nature in some cases. These models are interesting as they can be cited as specific examples of nonsingular (i.e., geometrically complete) inflationary universes. Also, it is worth mentioning here that entropy considerations favour the Einstein static model as the initial state for our universe $[6,7]$. Thereafter, a series of works [8-16] have been done to formulate emergent universe in different gravity models and also for various types of matter. Very recently, emergent scenario has been formulated with some interesting physical aspects. The idea of quantum tunneling [17] has been used for the decay of a scalar field having initial static state as false vacuum to a state of true vacuum. Secondly, a model of an emergent universe has been formulated in the background of nonequilibrium thermodynamical prescription with dissipation due to particle creation mechanism [18]. Very recently, Paul and Majumdar [19] have formulated emergent universe with interacting fields. Finally, Pavon et al. $[20,21]$ have studied the emergent scenario from thermodynamical view point. They have examined the validity of the generalized second law of thermodynamics 
during the transition from a generic initial Einstein static phase to the inflationary phase and also during the transition from inflationary era to the standard radiation dominated era.

\section{Chaplygin Gas and Possible Solution}

Mixed exotic fluid known as modified Chaplygin gas [21] has the equation of state $[22,23]$

$$
p=A \rho-\frac{B}{\rho^{n}}, \quad 0<n \leq 1 .
$$

This equation of state shows barotropic perfect fluid $p=A \rho$, at very early phase (when the scale factor $a(t)$ is vanishingly small), while it approaches $\Lambda C D M$ model when the scale factor is infinitely large. It shows a mixture at all stages. Note that at some intermediate stage the pressure vanishes and the matter content is equivalent to pure dust. Further, this typical model is equivalent to a self-interacting scalar field from field theoretic point of view. It should be noted that the Chaplygin gas was introduced in the context of aerodynamics. In the present paper, we will examine whether emergent scenario is possible for FRW model of the universe with matter content as modified Chaplygin gas (MCG).

For homogeneous and isotropic flat FRW model of the universe, the Einstein field equations are (choosing $8 \pi G=1$ )

$$
\begin{aligned}
& 3 H^{2}=\rho, \\
& 2 \dot{H}=-(\rho+p),
\end{aligned}
$$

with energy conservation relation:

$$
\dot{\rho}+3 H(\rho+p)=0
$$

Using (1) in (3), one can integrate $\rho$ as

$$
\rho=\left[\frac{B}{1+A}+\frac{c}{a^{3 \mu}}\right]^{1 /(n+1)},
$$

with $c>0$, a constant of integration.

Now, using this $\rho$ in the first Friedmann equation in (2), one can integrate to obtain cosmic time as a function of the scale factor as

$$
\begin{aligned}
& \frac{\sqrt{3}}{2}(1+A) c^{\alpha}\left(t-t_{0}\right) \\
& \quad=a^{3(1+A) / 2}{ }_{2} F_{1}\left[\alpha, \alpha, 1+\alpha,-\frac{B}{C(1+A)} a^{3(1+A) / 2 \alpha}\right],
\end{aligned}
$$

where $\alpha=1 / 2(1+n)$ and ${ }_{2} F_{1}$ is the usual hypergeometric function.

\section{Asymptotic Analysis and Equivalent Two Fluid Systems}

We will now analyze the two asymptotic cases. (i) When the Scale Factor " $a$ " Is Very Small. For small " $a$ ", $\rho$ can be approximated from (4) and $p$ can be approximated from (1) as follows:

$$
\begin{aligned}
\rho \cong & \left(\frac{\rho_{0}}{A+1}\right)^{1 /(n+1)} a^{-3(A+1)} \\
& +\frac{B}{(n+1)(A+1)^{1 /(n+1)} \rho_{0}^{(n /(n+1))}} a^{3(1+A) n} \\
\equiv & \rho_{1 i}+\rho_{2 i}, \\
p \cong & \frac{A \rho_{0}^{1 /(n+1)}}{(A+1)^{1 /(n+1)} a^{3(A+1)}} \\
& -\frac{B[1+n(A+1)]}{(n+1)(A+1)^{1 /(n+1)} \rho_{0}^{n /(n+1)}} a^{3 n(1+A)} \\
\equiv & p_{1 i}+p_{2 i} .
\end{aligned}
$$

(ii) When the Scale Factor " $a$ " Has Infinitely Large Value. Similarly, for large " $a$ ", $\rho$ and $p$ are approximated from (4) and (1), respectively, as follows:

$$
\begin{aligned}
\rho & \cong\left(\frac{B}{A+1}\right)^{1 /(n+1)}+\frac{\rho_{0}}{(n+1) B}\left(\frac{B}{A+1}\right)^{1 /(n+1)} a^{-3 \mu} \\
& \equiv \rho_{1 f}+\rho_{2 f}, \\
p & \cong-\frac{1}{(A+1)^{1 /(n+1)}}+\frac{n+(n+1) A}{(A+1)^{1 /(n+1)}} \frac{\rho_{0}}{(n+1) B} a^{-3 \mu} \\
& \equiv p_{1 f}+p_{2 f} .
\end{aligned}
$$

Thus, in the asymptotic limits, the components of energy density and pressure can be expressed as sum of two noninteracting barotropic fluids having equation of states:

$$
\begin{aligned}
w_{1 i} & =A, \\
w_{2 i} & =-[1+n(A+1)], \\
w_{1 f} & =-B^{-(1 /(n+1))}, \\
w_{2 f} & =\frac{n+(n+1) A}{B^{(1 /(n+1))}} .
\end{aligned}
$$

Thus, MCG can be considered in the asymptotic limits as two barotropic fluids of constant equation of state of which one is exotic in nature. However, one can consider that the two fluids in question (in the asymptotic limit) may be interacting with separate equation of state as

$$
\begin{gathered}
\dot{\rho_{1 i}}+3\left(\rho_{1 i}+p_{1 i}\right) H=Q_{i}, \\
\dot{\rho_{2 i}}+3\left(\rho_{2 i}+p_{2 i}\right) H=-Q_{i}, \\
\dot{\rho_{1 f}}+3\left(\rho_{1 f}+p_{1 f}\right) H=Q_{f}, \\
\rho_{2 f}+3\left(\rho_{2 f}+p_{2 f}\right) H=-Q_{f},
\end{gathered}
$$

where $Q_{i}$ and $Q_{f}$ represent the interaction term. 
$Q_{i}>0$ indicates a flow of energy from fluid 2 (having energy density $\rho_{2 i}$ ) to fluid 1 (having energy density $\rho_{1 i}$ ) and similarly for $Q_{f}$ also. Further, one can rewrite the conservation equations (9) as

$$
\begin{gathered}
\dot{\rho_{1 i}}+3 H\left(1+w_{1 i}^{\mathrm{eff}}\right) \rho_{1 i}=0, \\
\dot{\rho_{2 i}}+3 H\left(1+w_{2 i}^{\mathrm{eff}}\right) \rho_{2 i}=0, \\
\dot{\rho}_{1 f}+3 H\left(1+w_{1 f}^{\mathrm{eff}}\right) \rho_{1 f}=0, \\
\dot{\rho}_{2 f}+3 H\left(1+w_{2 f}^{\mathrm{eff}}\right) \rho_{2 f}=0,
\end{gathered}
$$

with

$$
\begin{aligned}
& w_{1 i}^{\mathrm{eff}}=w_{1 i}-\frac{Q_{i}}{3 H \rho_{1 i}}, \\
& w_{2 i}^{\mathrm{eff}}=w_{2 i}+\frac{Q_{i}}{3 H \rho_{2 i}}, \\
& w_{1 f}^{\mathrm{eff}}=w_{1 f}-\frac{Q_{f}}{3 H \rho_{1 f}}, \\
& w_{2 f}^{\mathrm{eff}}=w_{2 f}+\frac{Q_{f}}{3 H \rho_{2 f}} .
\end{aligned}
$$

The above conservation equations show that the fluids may be considered as noninteracting at the cost of variable equation of state.

\section{Emergent Scenario and Thermodynamical Analysis}

One should note that in integrating (3) to have (4) we assume that $A \neq-1$. Now, we will discuss the situation when $A=-1$.

The expression for energy density now becomes

$$
\rho=\left[3(n+1) B \ln \left(\frac{a}{a_{0}}\right)\right]^{1 /(n+1)},
$$

which from the first Friedmann equation gives

$$
\begin{aligned}
a=a_{0} \exp \left[b_{0}\left(t-t_{0}\right)^{(1 /(1-\alpha))}\right], & \\
b_{0} & =\left(\frac{\sqrt{3}}{2} B(2 n+1)\right)^{(1 /(1-\alpha))} .
\end{aligned}
$$

From the solutions (5) and (13), we see (Figures 1 and 2) that $a \rightarrow 0$ as $t \rightarrow-\infty$, so it is not possible to have emergent scenario with the usual modified Chaplygin gas. However, if we choose $-1<n<-1 / 2$, then $\alpha>1$ and we have from solution (13) $a \rightarrow a_{0}$ as $t \rightarrow-\infty$ (see Figure 3 ). Hence, it is possible to have emergent scenario with this revised form of MCG.

We will now discuss the thermodynamics of the emergent scenario with this revised form of MCG as the cosmic substratum.

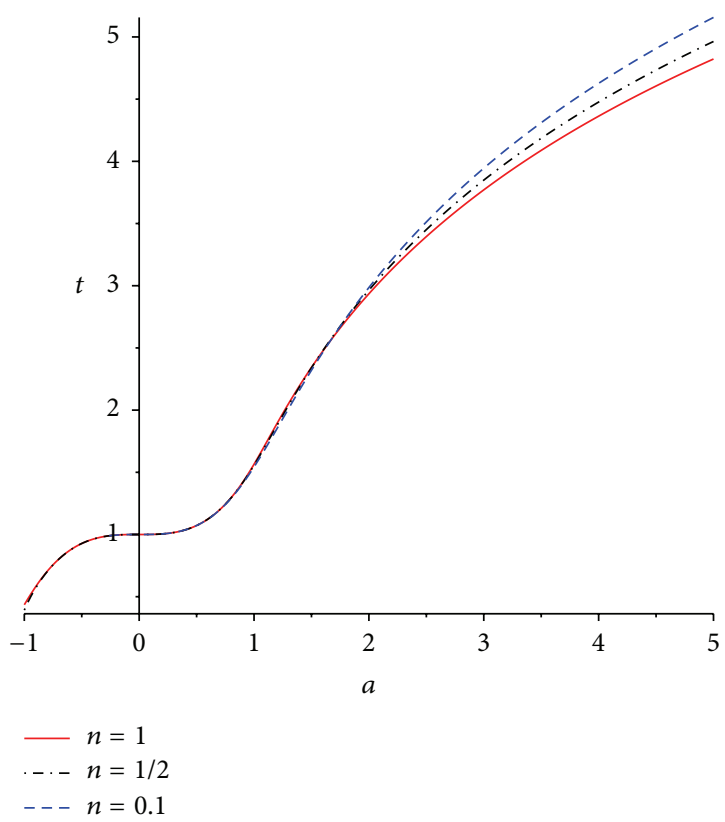

FIgure 1: The Figure represents the scale factor $a(t)$ against $t$ for $A \neq-1$.

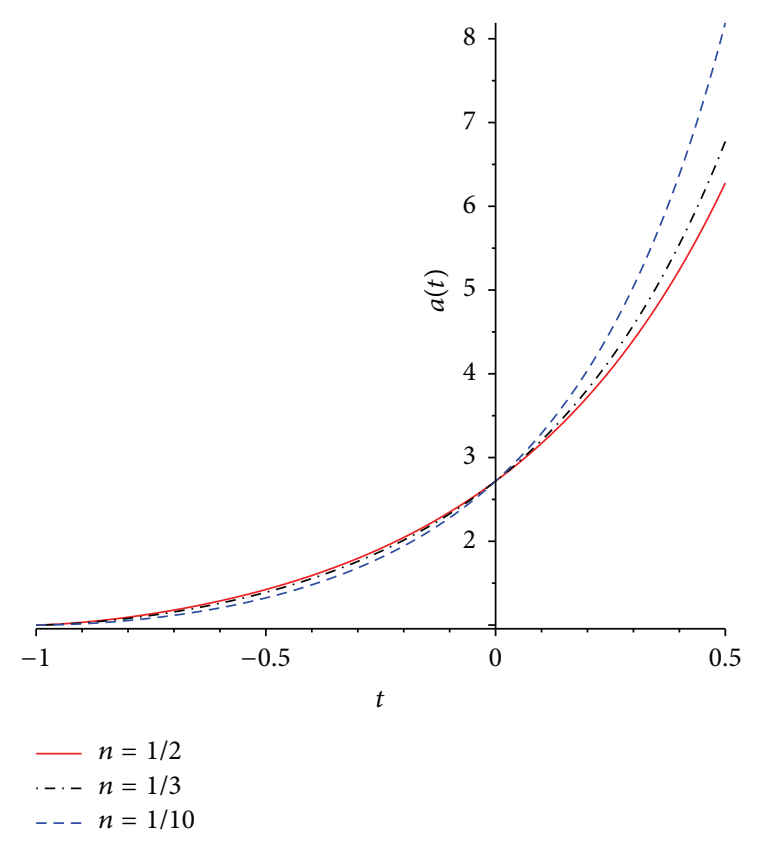

FIGURE 2: The Figure shows the graphical representation of the scale factor $a(t)$ against $t$ for $A=-1$.

Assuming the validity of the first law of thermodynamics at the horizon (having area radius $R_{h}$ ), we have the Clausius relation:

$$
-d E_{h}=T_{h} d S_{h},
$$

where $T_{h}$ is the temperature of the horizon and $s_{h}$ is the entropy of the horizon. In the above, $E_{h}$ is the amount of 


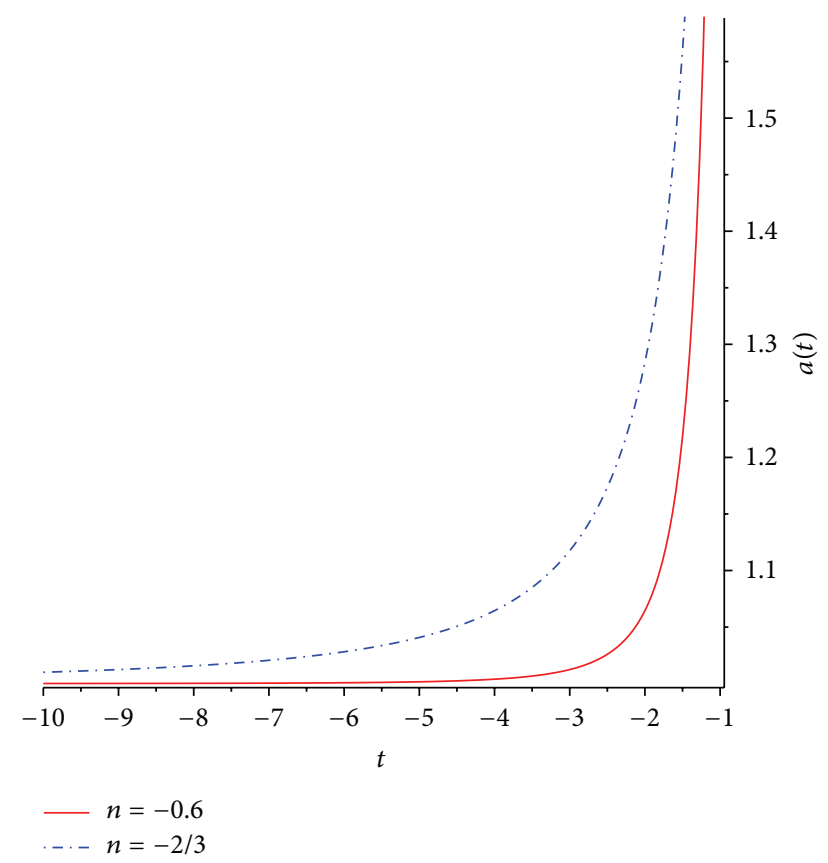

Figure 3: The Figure shows the graphical representation of the scale factor $a(t)$ against $t$ for $A=-1$.

energy crossing the horizon during time $d t$ and is given by [24-26]

$$
-d E_{h}=4 \pi R_{h}^{3} H(\rho+p) d t .
$$

So, using (15) in (14), we have the rate of change of the horizon entropy as

$$
\frac{d S_{h}}{d t}=\frac{4 \pi R_{h}^{3} H(\rho+p)}{T_{h}} .
$$

To obtain the entropy of the inside fluid, we start with the Gibbs equation [26, 27]

$$
T_{h} d S_{f}=d E_{f}+p d V
$$

where $S_{f}$ is the entropy of the fluid bounded by the horizon and $E_{F}$ is the energy of the matter distribution. Here, for thermodynamical equilibrium, the temperature of the fluid is taken as that of the horizon, that is, $T_{h}$.

Now, using $V=4 \pi R_{h}^{3} / 3, E_{f}=\left(4 \pi R_{h}^{3} / 3\right) \rho$, and the Friedmann equations, the entropy variation of the fluid is given by

$$
\frac{d S_{f}}{d t}=\frac{4 \pi R_{h}^{2}}{T_{h}}(\rho+p)\left(\dot{R_{h}}-H R_{h}\right) .
$$

Thus, combining (16) and (18), the variation of the total entropy $\left(S_{T}\right)$ is given by

$$
\frac{d S_{T}}{d t}=\frac{d}{d t}\left(S_{h}+S_{f}\right)=\frac{4 \pi R_{h}^{2}}{T_{h}}(\rho+p) \dot{R_{h}} .
$$

Case 1 (apparent horizon). The area radius for apparent horizon is given by

$$
R_{A}=\frac{1}{H}
$$

so that

$$
\dot{R}_{A}=-\frac{\dot{H}}{H^{2}}=\frac{4 \pi G(\rho+p)}{H^{2}} .
$$

Hence,

$$
\frac{d S_{T}}{d t}=\frac{(4 \pi)^{2} G(\rho+p)^{2}}{T_{A} H^{4}}>0 .
$$

Thus, generalised second law of thermodynamics (GSLT) is always true at the apparent horizon.

Case 2 (event horizon). The area radius for event horizon is given by

$$
R_{E}=a \int_{t}^{\infty} \frac{d t}{a}
$$

The above improper integral converges for accelerating phase of the FRW model. Hence, in the present scenario, it is very much relevant. From the above definition

$$
\dot{R}_{E}=H R_{E}-1 \text {, }
$$

so from (19)

$$
\begin{aligned}
\frac{d S_{T}}{d t} & =\frac{(4 \pi) R_{E}^{2}(\rho+p)}{T_{E}}\left(H R_{E}-1\right) \\
& =\frac{(4 \pi) R_{E}^{2} H}{T_{E}}\left[(A+1) \rho-\frac{B}{\rho^{n}}\right]\left(R_{E}-R_{A}\right) \\
& =\frac{(4 \pi) R_{E}^{2} H}{T_{E}} \frac{c(1+a)}{a^{3 \mu} \cdot \rho^{n}}\left(R_{E}-R_{A}\right) .
\end{aligned}
$$

Hence, the validity of GSLT is possible if $R_{E}>R_{A}$ (as $\alpha>1$ ). In the above, temperature is chosen as the hawking temperature on the horizon as $[28,29]$

$$
\begin{aligned}
& T_{A}=\frac{1}{2 \pi R_{A}}, \\
& T_{E}=\frac{R_{E}}{2 \pi R_{A}^{2}} .
\end{aligned}
$$

\section{Additional Points}

In the present work, we have examined the cosmology of the emergent scenario for modified Chaplygin gas as the cosmic fluid. It is found that for both the solutions (with $A \neq-1$ and $A=-1$ ) the model does not exhibit emergent scenario at early epochs. So, one can conclude that it is not possible to have emergent scenario with MCG. However, if $n$ is chosen to be negative, that is, $-1<n<-1 / 2$, then $a \rightarrow a_{0}$ as $t \rightarrow-\infty$; that is, initial big bang singularity is avoided.

Finally, thermodynamical analysis of the emergent scenario has been presented. 


\section{Competing Interests}

The authors declare that they have no competing interests.

\section{Acknowledgments}

Subenoy Chakraborty thanks Inter-University Center for Astronomy and Astrophysics (IUCAA), Pune, India, for their warm hospitality as a part of the work was done during a visit. Also Subenoy Chakraborty thanks UGC-DRS programme at the Department of Mathematics, Jadavpur University. Sourav Dutta thanks the Department of Science and Technology (DST), Government of India, for awarding Inspire research fellowship.

\section{References}

[1] E. R. Harrison, "Classification of uniform cosmological models," Monthly Notices of the Royal Astronomical Society, vol. 137, no. 1, pp. 69-79, 1967.

[2] G. F. Ellis and R. Maartens, "The emergent universe: inflationary cosmology with no singularity," Classical and Quantum Gravity, vol. 21, no. 1, pp. 223-232, 2004.

[3] G. F. R. Ellis, J. Murugan, C. G. Tsagas et al., "The emergent universe: an explicit construction," Classical and Quantum Gravity, vol. 21, no. 1, p. 233, 2004.

[4] S. Mukherjee, B. C. Paul, S. D. Maharaj, and A. Beesham, "Emergent universe in starobinsky model," http://arxiv.org/abs/gr-qc/ 0505103.

[5] S. Mukherjee, B. C. Paul, N. K. Dadhich, S. D. Maharaj, and A. Beesham, "Emergent universe with exotic matter," Classical and Quantum Gravity, vol. 23, no. 23, pp. 6927-6934, 2006.

[6] G. W. Gibbons, "The entropy and stability of the universe," Nuclear Physics B, vol. 292, no. 4, pp. 784-792, 1987.

[7] G. W. Gibbons, "The entropy and stability of the universe," Nuclear Physics B, vol. 292, pp. 784-792, 1987.

[8] D. J. Mulryne, R. Tavakol, J. E. Lidsey, and G. F. R. Ellis, “An emergent universe from a loop," Physical Review D, vol. 71, no. 12, Article ID 123512, 2005.

[9] A. Banerjee, T. Bandyopadhyay, and S. Chakraborty, "Emergent Universe in the brane-world scenario," General Relativity and Gravitation, vol. 40, no. 8, pp. 1603-1607, 2008.

[10] A. Banerjee, T. Bandyopadhyay, and S. Chakraborty, "Emergent universe in brane world scenario with Schwarzschild-de Sitter bulk," General Relativity and Gravitation, vol. 40, no. 8, pp. 1603-1607, 2008.

[11] N. J. Nunes, "Inflation: a graceful entrance from loop quantum cosmology," Physical Review D, vol. 72, no. 10, Article ID 103510, 2005.

[12] J. E. Lidsey and D. J. Mulryne, "Graceful entrance to braneworld inflation," Physical Review D, vol. 73, no. 8, Article ID 083508, 2006.

[13] U. Debnath, "Emergent universe and the phantom tachyon model," Classical and Quantum Gravity, vol. 25, no. 20, Article ID 205019, 8 pages, 2008.

[14] B. C. Paul and S. Ghose, "Emergent universe scenario in the Einstein-Gauss-Bonnet gravity with dilaton," General Relativity and Gravitation, vol. 42, no. 4, pp. 795-812, 2010.

[15] U. Debnath and S. Chakraborty, "Emergent universe with exotic matter in brane world scenario," International Journal of Theoretical Physics, vol. 50, no. 9, pp. 2892-2898, 2011.
[16] S. Mukerji, N. Mazumder, R. Biswas, and S. Chakraborty, "Emergent scenario and different anisotropic models," International Journal of Theoretical Physics, vol. 50, no. 9, pp. 2708-2719, 2011.

[17] P. Labraña, "Emergent universe by tunneling," Physical Review $D$, vol. 86, no. 8, Article ID 083524, 2012.

[18] S. Chakraborty, "Is emergent universe a consequence of particle creation process?” Physics Letters B, vol. 732, pp. 81-84, 2014.

[19] B. C. Paul and A. Majumdar, "Emergent universe with interacting fluids and the generalized second law of thermodynamics," Classical and Quantum Gravity, vol. 32, no. 11, Article ID 115001, 2015.

[20] S. D. Campo, R. Herrera, and D. Pavon, "The generalized second law in the emergent universe," Physics Letters B, vol. 707, no. 1, pp. 8-10, 2012.

[21] D. Pavon, S. del Campo, and R. Herrera, "The generalized second law and the emergent universe," http://arxiv.org/abs/1212 .6863 .

[22] U. Debnath, A. Banerjee, and S. Chakraborty, "Role of modified Chaplygin gas in accelerated universe," Classical and Quantum Gravity, vol. 21, no. 23, pp. 5609-5617, 2004.

[23] H. Benaoum, "Accelerated universe from modified chaplygin gas and tachyonic fluid," http://arxiv.org/abs/hep-th/0205140.

[24] R.-G. Cai and S. P. Kim, "First law of thermodynamics and Friedmann equations of Friedmann-Robertson-Walker universe," Journal of High Energy Physics, vol. 2005, no. 2, article 050, 2005.

[25] R. S. Bousso, "Cosmology and the S matrix," Physical Review D, vol. 71, no. 6, Article ID 064024, 2005.

[26] N. Majumder and S. Chakraborty, "Does the validity of the first law of thermodynamics imply that the generalized second law of thermodynamics of the universe is bounded by the event horizon?" Classical and Quantum Gravity, vol. 26, no. 19, Article ID 195016, 2009.

[27] G. Izquierdo and D. Pavón, "Dark energy and the generalized second law," Physics Letters B, vol. 633, no. 4-5, pp. 420-426, 2006.

[28] S. Chakraborty, "Is thermodynamics of the universe bounded by event horizon a Bekenstein system?" Physics Letters B, vol. 718, no. 2, pp. 276-278, 2012.

[29] S. Saha and S. Chakraborty, "A redefinition of Hawking temperature on the event horizon: thermodynamical equilibrium," Physics Letters B, vol. 717, no. 4-5, pp. 319-322, 2012. 

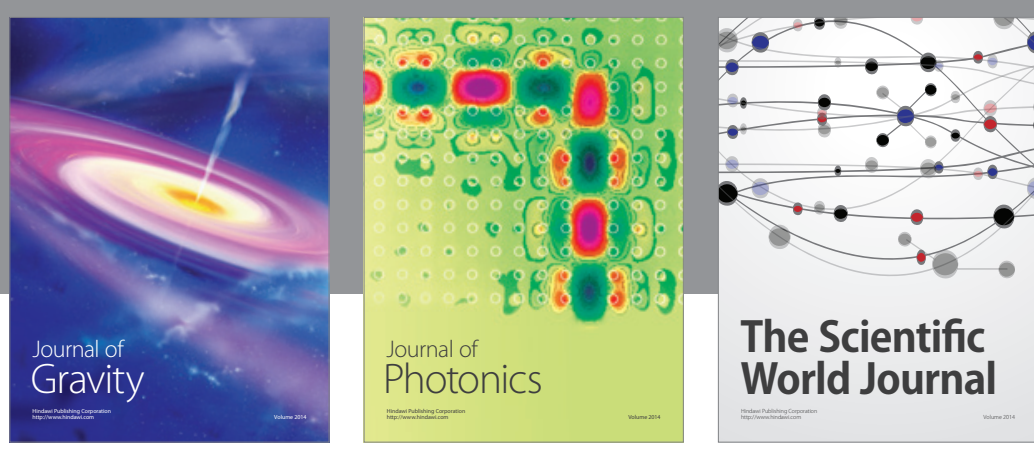

The Scientific World Journal
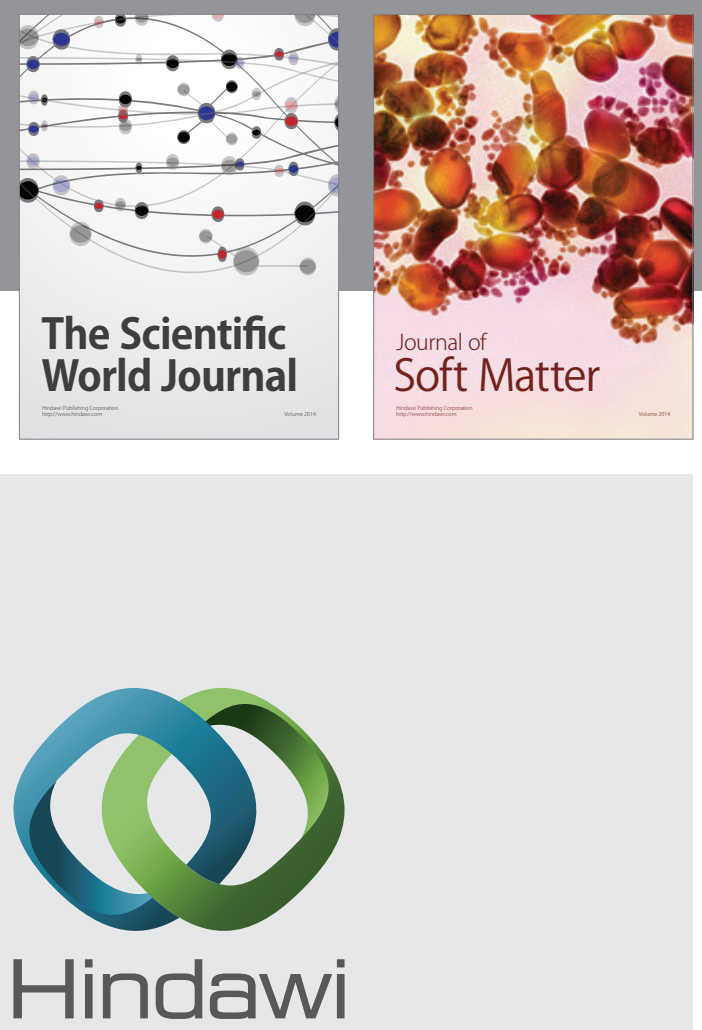

Submit your manuscripts at

http://www.hindawi.com

nternational Journal of

Statistical Mechanics
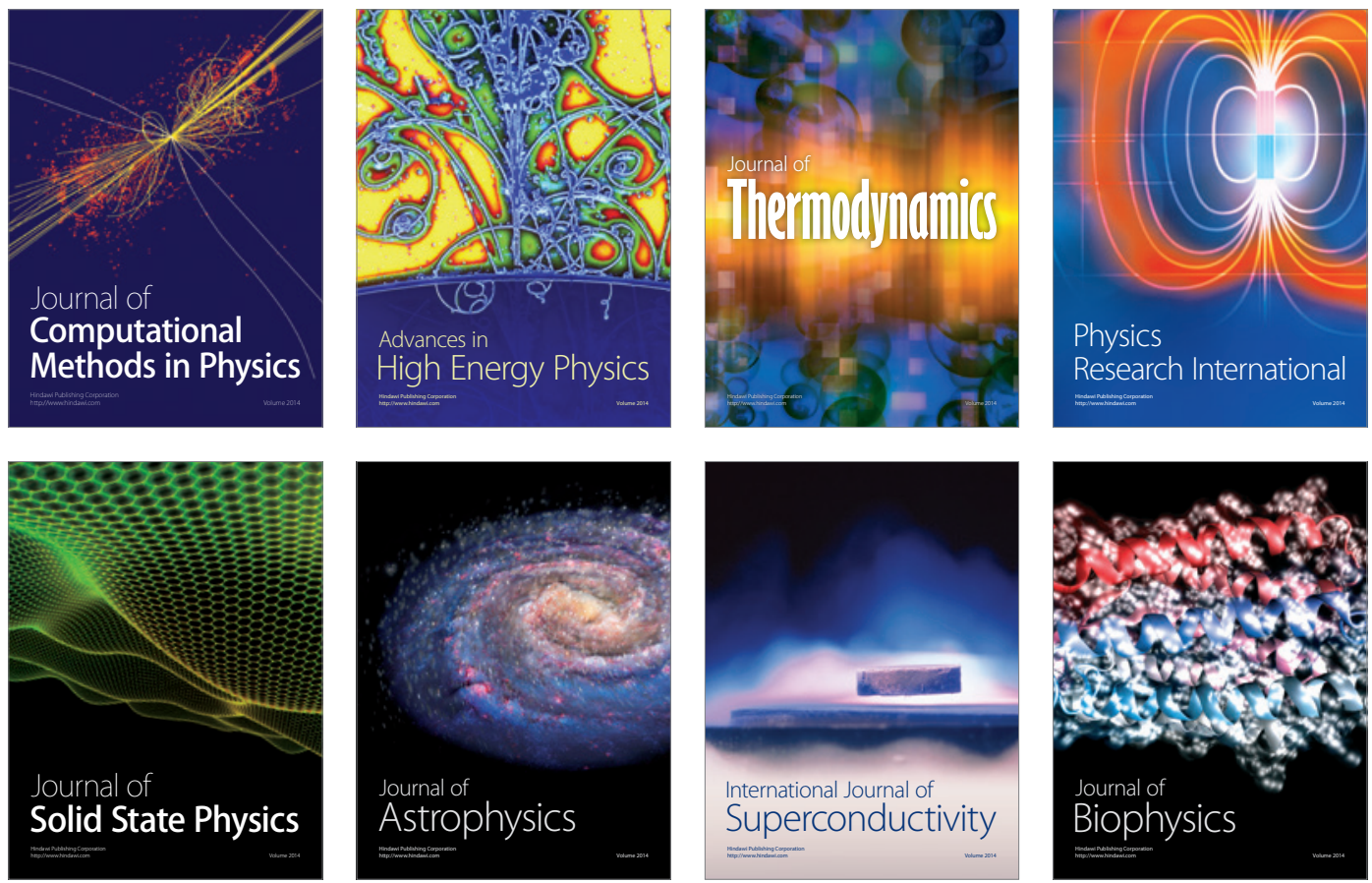
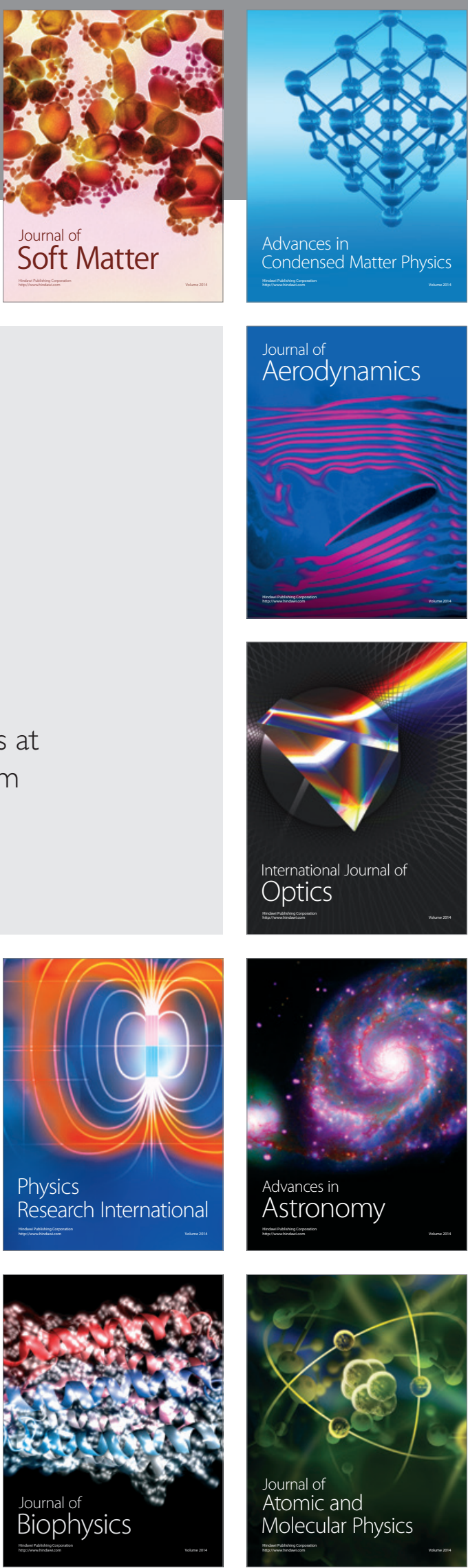